\title{
Microfluidic wound bandage: Localized oxygen modulation of collagen maturation
}

\author{
Joe F. Lo, PhD',2,3; Martin Brennan, BS3; Zameer Merchant, BS ; Lin Chen, MD, PhD²; Shujuan Guo, PhD²; \\ David T. Eddington, $\mathrm{PhD}^{3}$; Luisa A. DiPietro, DDS, $\mathrm{PhD}^{2}$ \\ 1. Department of Mechanical Engineering, College of Engineering and Computer Sciences, University of Michigan at Dearborn, Dearborn, \\ Michigan, \\ 2. Center for Wound Healing and Tissue Regeneration, Department of Periodontics, College of Dentistry, and \\ 3. Department of Bioengineering, College of Engineering, University of Illinois at Chicago, Chicago, Illinois
}

\author{
Reprint requests: \\ Professor J. F. Lo, Mechanical Engineering \\ Department, University of \\ Michigan-Dearborn, 4901 Evergreen Road, \\ 1340 HPEC, Dearborn, MI 48128-1491, \\ USA. \\ Tel: +1 3135930913 \\ Fax: +1 313593 3851; \\ Email: jfjlo@umd.umich.edu
}

Manuscript received: January 31, 2012

Accepted in final form: November 29 ,

2012

DOI:10.1111/wrr.12021

\begin{abstract}
Restoring tissue oxygenation has the potential to improve poorly healing wounds with impaired microvasculature. Compared with more established wound therapy using hyperbaric oxygen chambers, topical oxygen therapy has lower cost and better patient comfort, although topical devices have provided inconsistent results. To provide controlled topical oxygen while minimizing moisture loss, a major issue for topical oxygen, we have devised a novel wound bandage based on microfluidic diffusion delivery of oxygen. In addition to modulating oxygen from 0 to $100 \%$ in 60 seconds rise time, the microfluidic oxygen bandage provides a conformal seal around the wound. When $100 \%$ oxygen is delivered, it penetrates wound tissues as measured in agar phantom and in vivo wounds. Using this microfluidic bandage, we applied the oxygen modulation to $8 \mathrm{~mm}$ excisional wounds prepared on diabetic mice. Treatment with the microfluidic bandage demonstrated improved collagen maturity in the wound bed, although only marginal differences were observed in total collagen, microvasculature, and external closure rates. Our results show that proper topical oxygen can improve wound parameters underneath the surface. Because of the ease of fabrication, the oxygen bandage represents an economical yet practical method for oxygen wound research.
\end{abstract}

Oxygen is pervasive in wound healing processes and plays a number of critical roles in inflammation, proliferation, and tissue remodeling phases. During the initial inflammatory phase, oxygen modulation in the forms of reactive oxygen species and hypoxia signals leukocyte recruitment ${ }^{1-3}$ and stimulates macrophage secretion of angiogenic factors, ${ }^{4-6}$ respectively. Hypoxia has been demonstrated to promote keratinocyte migration, ${ }^{7,8}$ the key process in reepithelialization. Conversely, oxygen is critical to many steps of healing. During the proliferative phase, oxygen promotes proliferation and migration in fibroblast and endothelial cells, ${ }^{9-11}$ and stimulates fibroblasts to lay down collagen to strengthen the wound. ${ }^{12,13}$ Moreover, collagen maturation during the remodeling phase is an oxygen-intensive process. ${ }^{12,14}$ Disturbances of these oxygen dynamics are implicated in poorly healing wounds, including those found in diabetic individuals. Diabetic wounds with poor microvasculature and thus inadequate tissue oxygenation have impaired fibroblast, endothelial proliferation and function. ${ }^{15-17}$ These wounds show prolonged inflammation, poor angiogenesis, and impaired collagen maturity, ${ }^{18-20}$ leading to delayed closure and recurrence due to inadequate strength of underlying tissue. ${ }^{21}$ Restoration of proper oxygen dynamics has been suggested as a therapy to improve the speed and maturity of healing in these wounds.

The most common therapeutic techniques to restore wound oxygenation are the Hyperbaric Oxygen Therapy (HBOT) and the Topical Oxygen Therapy (TOT). HBOT involves an established protocol derived from hyperbaric chamber usage in gas poisoning and decompression sickness, with clear mechanisms of tissue oxygenation. ${ }^{22,23}$ However, HBOT subjects the patient in a sealed and pressurized ( 2 to $3 \mathrm{~atm}$ ) oxygen environment, risking claustrophobia, ear damage, and systemic neurotoxicity and oxidative damage. ${ }^{24} \mathrm{TOT}$, on the other hand, is directly applied to the surface of the wound at atmospheric pressure, typically through continuous oxygen flow in a bladder device. ${ }^{25-28}$ TOT improves the cost, convenience, and patient comfort during the wound therapy. However, gas flow from TOT device convectively dries the wound, a situation that might inhibit further oxygen diffusion. ${ }^{29}$ Hence, creating a topical oxygen technique with moisture control might improve oxygen delivery. Another limitation of HBOT and TOT is that these methods cannot achieve rapid gas level modulation due to the large amount of gas volume to be exchanged. Both HBOT and TOT in their current forms deliver a constant level of oxygen, e.g., 100\% without the temporal dynamics observed in real wounds. The development of miniature oxygen bandages that allow controlled oxygen delivery with rapid adjustment and moisture control may resolve the therapeutic limitations of HBOT and TOT in the treatment of wounds.

To demonstrate controlled oxygen therapy without convective drying, we employed microfluidic control of oxygen similar to previously demonstrated techniques of modulating oxygen via microfluidic gas channels. ${ }^{30-32}$ Using this technology, we designed a microfluidic bandage that delivers oxygen to the wound via diffusion only. The bandage, shown in 


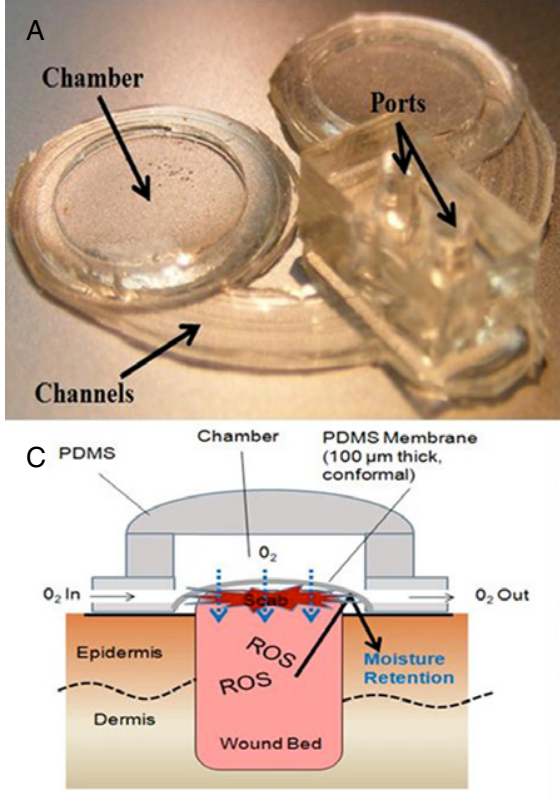

Schematic (cross-section)
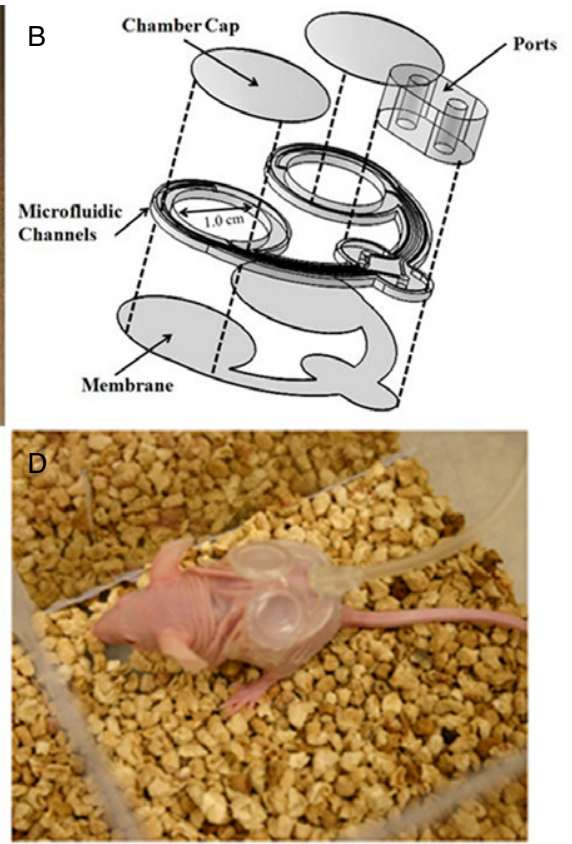

Figure 1. Microfluidic oxygen bandage: (A) the microfluidic component of the bandage is entirely PDMS, (B) the microfluidics are comprised of multiple layers, including microfluidic channels, $10 \mathrm{~mm}$ chambers with dome caps, and a gas-permeable membrane, (C) diffusion via this membrane provides oxygen delivery without convective flow, (D) up to six animals can be treated simultaneously by mounting the oxygen bandages with Tegaderm and tethering them to a medical grade oxygen tank.
Figure 1, is comprised of a microfluidic component embedded in Tegaderm (3M, St. Paul MN) dressing adhesive. The microfluidic component, fabricated from polydimethylsiloxane (PDMS), features (1) $300 \mu \mathrm{m}$ wide microfluidic channels, (2) two oxygen-filled chambers $10 \mathrm{~mm}$ in diameter, and (3) $100 \mu \mathrm{m}$ thick gas-permeable PDMS membrane. The bandage is designed to be connected to medical grade oxygen tanks through the microchannels, filling up the oxygen chambers and diffusing oxygen through the elastic membranes directly to the wound. The elastic bandage provides localized oxygen delivery that conforms to skin topology while minimizing convective drying. Furthermore, the construction of a sixcelled cage allowed the simultaneous treatment of multiple tethered animals while allowing them to roam freely (Figure 1). Having an easy-to-apply oxygen bandage combined with the ability to do treatments on multiple animals represents a novel and useful technology for studying oxygen effects in wound healing. With this goal in mind we demonstrate here the design, fabrication, and validation of such microfluidic bandage for studying diabetic wound healing in mice.

\section{MATERIALS AND METHODS}

\section{Microfluidic oxygen bandage}

The microfluidic component of the oxygen bandage is a multilayer device comprised of a dome encapsulation layer, a microfluidic layer, and a PDMS membrane layer from top to bottom, shown in Figure 1B. The dome cap was formed by pipetting $250 \mu \mathrm{L}$ of premixed PDMS and dropping onto a silicon wafer heated to $120^{\circ} \mathrm{C}$, allowing the droplet to form a semispherical section before curing. The microfluidic channels were patterned by exposing UV light through a computer-designed photomask (FineLine Imaging, Colorado
Springs, CO) onto $300 \mu \mathrm{m}$ thick SU8 photoresist, and developing away the unexposed material. This pattern was transferred by pouring PDMS prepolymer over the SU8 and cured at $90{ }^{\circ} \mathrm{C}$ for 2 hours. The membrane layer was made by spinning PDMS on another silicon wafer at $800 \mathrm{rpm}$ for 30 seconds, then curing at $85{ }^{\circ} \mathrm{C}$ for 5 minutes. After making all three layers, appropriate access ports and $10 \mathrm{~mm}$ chambers were punched, and the surface was treated with a corona plasma device (Electro-Technic Products, Inc., Chicago, IL) for 30 seconds prior to bonding. Aligned and bonded devices were baked at $120{ }^{\circ} \mathrm{C}$ for 2 hours to further strengthen the bonding. Finished devices were submerged under a water bath and leak tested by flowing air through the device with a syringe.

\section{Oxygen bandage validations}

To test the controlled, localized delivery of oxygen, the bandages were placed against a fluorescence oxygen sensor and imaged under an Olympus IX71 microscope. The oxygen sensor was coated with ruthenium dye, whose fluorescence is quenched by oxygen (Foxy-slides, Ocean Optics, Inc, Dunedin, FL). Ten, 21, 60, and $100 \%$ oxygen was supplied to the bandage, and the final diffused oxygen was measured over time. To simulate proper sealing of oxygen delivery over wound topology, a gas-permeable obstacle $(5 \mathrm{~mm}$ round, $200 \mu \mathrm{m}$ tall PDMS dome cap) was tested underneath two types of bandages: a bandage with deformable membrane and a bandage with rigidly supported membrane. Diffused oxygen was measured underneath the obstacles and compared. Lastly, oxygen penetration was tested with $3 \%$ agar gel, to simulate skin diffusion. These phantom tissues were cut to 0.2 to $1 \mathrm{~mm}$ thicknesses in $0.1 \mathrm{~mm}$ increments with a Vibratome (Series 1000 Classic, Ted Pella, Inc., Redding, CA) and sandwiched between the bandage and sensor for characterization. 
Table 1. SKH1 mice groups prior to wound healing experiments

\begin{tabular}{lccc}
\hline & Normal & Diabetic & Diabetic $+\mathrm{O}_{2}$ \\
\hline$n$ & 5 & 5 & 5 \\
Weight & $24.5+3.1 \mathrm{~g}$ & $25.2+1 \mathrm{~g}$ & $24.0+0.8 \mathrm{~g}$ \\
Glucose & $73.5 \pm 9.9 \mathrm{mg} / \mathrm{dL}$ & $287 \pm 69 \mathrm{mg} / \mathrm{dL}$ & $275 \pm 17 \mathrm{mg} / \mathrm{dL}$ \\
\hline
\end{tabular}

Errors are standard deviations.

\section{Animal protocol}

All animal procedures followed University of Illinois guidelines for the humane treatment of animals and were approved by the University of Illinois Institutional Animal Care and Use Committee. To test the effects of microfluidic oxygen on wounds, SKH1 hairless mice (Charles River Laboratories, Wilmington, MA) were used, taking advantage of their skin morphology for easy wound bandaging. Three groups of normal, diabetic, and oxygen-treated diabetic animals were used, as listed in Table 1. The last two groups were injected with low-dose streptozotocin (STZ) at $50 \mathrm{mg} / \mathrm{kg}$ daily for 5 consecutive days, following detailed protocols at http:// www.diacomp.org. Diabetic animals were verified with a tail pinch glucose test, at $200 \mathrm{mg} / \mathrm{dL}$ cutoff. Animals that did not achieve this glucose level were treated again 7 weeks after the first injection. The combined STZ induction rate was $80 \%$.

All animals were 13 weeks old at the time of wounding. Animals were anesthetized with $100 / 5 \mathrm{mg} / \mathrm{kg}$ ketamine/ xylaxine. The backs of the animals were cleaned with an isopropyl alcohol wipe. Skin from the back was folded against a rubber disk and wounds were prepared with an $8 \mathrm{~mm}$ circular biopsy punch, resulting in two full thickness wounds at $15 \mathrm{~mm}$ distance apart center to center. Oxygen bandages, with or without oxygen flow, were fitted on all animals prior to their waking. The wounds were followed for 14 days after wounding. Pictures of the physical wounds were taken each day before treatment. Animals were sacrificed at day 14 after wounding, and tissue samples were used to prepare frozen histologic section, paraffin embedded histologic section, and collagen analysis via quantification of hydroxyproline.

\section{Oxygen treatment protocol}

All three animal groups were fitted with bandages, with $100 \%$ oxygen tethered only to the treatment group. The other two groups were in equilibrium with ambient air (21\% oxygen) and regarded as nondiabetic and diabetic controls. For the treatment group, a six-celled cage was constructed for simultaneous treatment of the five animals, as shown in Figure 1D. Plexiglass was cut and silicone-glued as divider inserts in a $2^{\prime} \times 1.5^{\prime} \times 1^{\prime}$ transparent plastic box. The lid was cut with windows to allow tethered gas lines to access animals in the cells. Food and water was supplied to each animal directly and via a $15 \mathrm{~mL}$ test tube respectively. Gas lines were tethered to the animals with a $360^{\circ}$ freely rotating quick connect coupler (Colder Products, St. Paul, MN). All tethered lines were supplied through a flow adjustable manifold connected to a single silicone line hooked up to a $697 \mathrm{~L}$ medical-grade $100 \%$ oxygen tank. Oxygen was supplied to the diabetic treatment group 4 hours a day for 14 days starting immediately after wounding. At the end of each treatment day, bandages from all animal groups were removed, boiled for 5 minutes in water, disinfected with alcohol wipes, and tested for leakage in preparation for use the next day. Broken or leaky devices were replaced with new ones.

\section{In vivo tissue oxygen penetration quantification}

To quantify the extent of wound oxygen penetration, we used a fiber optic fluorescence oxygen probe (FOXY Probe, Ocean Optics) to measure oxygen tension. This method allows direct measurement of tissue oxygen penetrations across the entire skin/wound thickness noninvasively. By inserting the fiber optic probe beneath the skin and measuring before and after $100 \%$ oxygen was introduced, we recorded an elevated oxygen tensions above the baseline. Days 3, 7, and 14 after wounding were included as measurement time points.

\section{Tissue tests and histology}

\section{Hydroxyproline analysis}

The measurement of hydroxyproline is commonly used as a relevant marker of total collagen content. Both normal and wound tissues were harvested at day 14 . Tissue samples were weighed and hydrolyzed in $6 \mathrm{M} \mathrm{HCl}$ immediately after collection and analyzed for hydroxyproline using a colorimetric kit from QuickZyme Biosciences (Leiden, The Netherlands). Standard solutions from 12.5 to $300 \mu \mathrm{M}$ were included in the assay, and samples were read in duplicates on a 96-well plate at $570 \mathrm{~nm}$ in a plate reader (Molecular Devices, Sunnyvale, CA).

\section{Histologic assessment}

To examine tissue vascularity, frozen sections were subjected to immunohistochemical staining for CD31, a marker of blood vessels, as previously described. ${ }^{33}$ Briefly $7 \mu \mathrm{m}$ sections were cut from Optimal Cutting Temperature Compound embedded specimens (Sakura Finetek USA Inc., Torrance, $\mathrm{CA}$ ), and then fixed in $-20^{\circ} \mathrm{C}$ acetone for 10 minutes. Sections were blocked with $10 \%$ goat serum for 30 minutes, stained with primary rat anti-mouse CD31 (BD Pharmingen, $0.31 \mu \mathrm{g} / \mathrm{mL}$ ) for 45 minutes, incubated with secondary Alexa 594 goat anti-rat IgG $(2 \mu \mathrm{g} / \mathrm{mL}$, Life Technologies, Grand Island, NY) for 45 minutes in the dark, and mounted in 50\% glycerol on a microscope slide. CD31 positive staining under fluorescent microscopy was quantified using Image J software (National Institutes of Health, Bethesda, MD). For picrosirius red staining, paraffin embedded samples were cut to $5 \mu \mathrm{m}$ thick, rehydrated, treated with phosphomolybdic acid at 


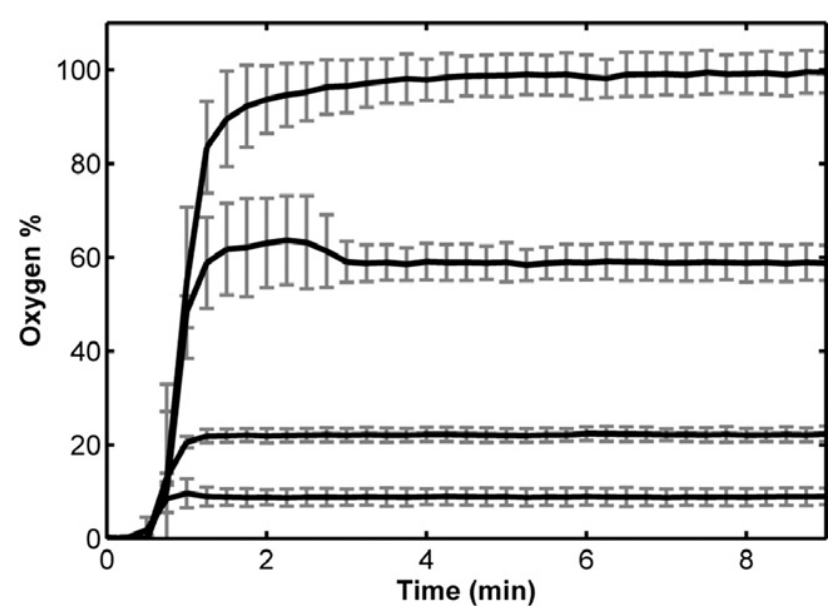

Figure 2. Multiple concentrations of oxygen-10, 21, 60, and $100 \%$ - can be delivered by the microfluidic oxygen bandage, with time scales less than 60 seconds. Error bars denote standard deviations, $n=3$ experiments.

$0.2 \%$, stained with picrosirius red solution for 90 minutes, washed in $70 \%$ ethanol, dehydrated and mounted in xylene on a microscope slide. Picrosirius staining images were viewed under polarized microscopy and quantified by dividing the areas of the red fibers by those of the green, using automatic measurement programs provided by Axio Vision software (Carl Zeiss, Thornwood, NY).

\section{Data analysis and statistics}

For device characterization, $n=3$ experiments were conducted for Figures 2-5, in which errors were expressed as standard deviations. For the animal experiments, each animal produced two wounds; and each wound was cut directly in half, producing four tissues, of which three were randomized for the hydroxyproline, CD31, and picrosirius tests. For each test, $n=5$ wounds from different animals were used for each condition in Figures 6-9, in which errors were expressed as standard deviations. Individual analyses of variance
(ANOVA) were performed first to assess the group difference. Post hoc pairwise comparisons by individual-samples $t$-test (single sided) were then examined. Please note that multiple comparisons adjustments were not performed for these analyses, but the $p$-values and $95 \%$ confidence intervals (CI) are provided for interpretation. ${ }^{34}$

\section{RESULTS}

\section{Animal glucose levels}

Glucose levels of all three animal groups were monitored during STZ induction and measured again prior to wounding. All mice exhibited similar body weights of about 24-25 g. The fasting glucose levels of normal animals were $73.5 \pm 9.9 \mathrm{mg} / \mathrm{dL}$; levels for untreated and treated diabetic mice measured $287 \pm 69 \mathrm{mg} / \mathrm{dL}$ and $275 \pm 17 \mathrm{mg} / \mathrm{dL}$, respectively. Following the STZ diabetes induction, animals with glucose $<200 \mathrm{mg} / \mathrm{dL}$ were not used for experiments. Table 1 shows the number and characteristics of animals used in the experiments.

\section{Modulation of oxygen concentrations}

The microfluidic oxygen delivery through the PDMS membrane was characterized by perfusing the microfluidic channels with gases $(10,21,60$ or $100 \%$ oxygen) as shown in Figure 2. Equilibration of all oxygen concentrations were achieved in less than 1 minute.

\section{Localization of oxygen delivery in nonconformal and conformal devices}

To demonstrate proper sealing of the oxygen bandage, the conformal delivery of oxygen was characterized by diffusing oxygen through a predefined obstacle (PDMS, $5 \mathrm{~mm}$ round by $200 \mu \mathrm{m}$ tall) underneath two types of oxygen bandages, as shown in Figure 3A and B. One device had support channels $100 \mu \mathrm{m}$ tall, referred to here as nonconformal bandage. The other had a much taller chamber of $1 \mathrm{~mm}$ thick without support pillars, allowing the membrane to deform around the
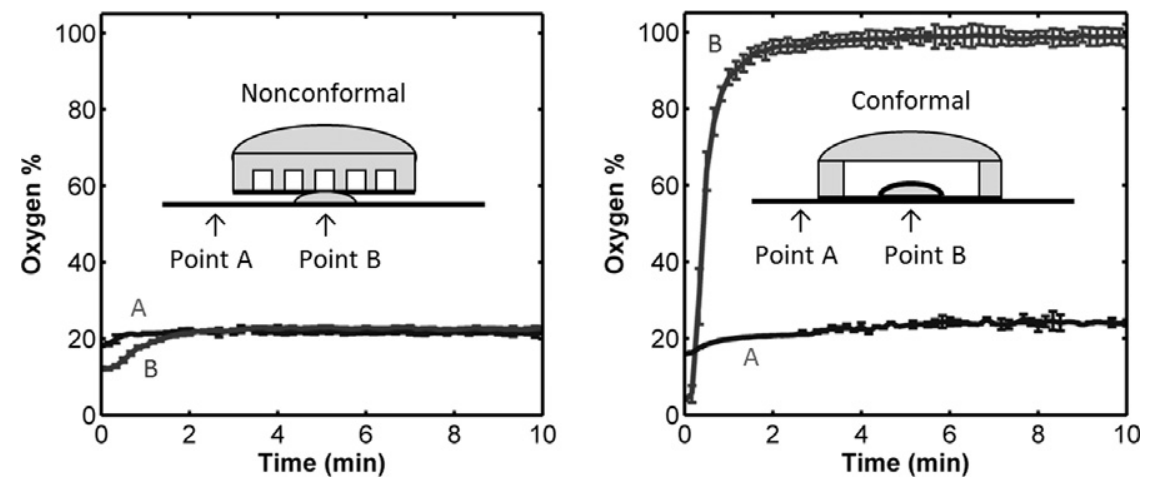

Figure 3. Oxygen delivery via conformal bandage can provide better delivery to a rough surface. Line $A$ (blue) represents background measurements external (point A) to the oxygen chamber while line $B$ (red) represents position (point B) directly underneath the obstacle. (A) Rigid membrane device cannot provide sealing around an obstacle ( $5 \mathrm{~mm}$ round, $200 \mu \mathrm{m}$ tall), and cannot deliver oxygen through the obstacle, (B) Elastic membrane in oxygen bandage seals around the obstacle and provides 100\% oxygen delivery through the obstacle. Error bars denote standard deviations, $n=3$ experiments. 




Figure 4. Oxygen delivered by bandage can penetrate agar. After 10 minutes of equilibration, oxygen can be elevated up to $80 \%$ across a $0.8 \mathrm{~mm}$ thick section of agar tissue phantom. Error bars denotes standard deviations of three agar samples.

obstacle and referred to here as conformal bandage. For the nonconformal bandage, the delivered $100 \%$ oxygen did not increase the concentration underneath the obstacle, which stayed close to background ambient levels at $22.61 \pm 0.44 \%$ $\mathrm{O}_{2}$. On the other hand, the conformal device delivered and raised the oxygen directly underneath the obstacle to $99.5 \pm 4.40 \% \mathrm{O}_{2}$ at equilibrium. These results showed that the conformal device provided better sealing around a rough surface similar to normal wound topology.

\section{Microfluidic oxygen penetration}

The penetration of microfluidic oxygen delivery through thicknesses of $3 \%$ agar phantom tissue $(0.2-1.0 \mathrm{~mm}$ in 0.1

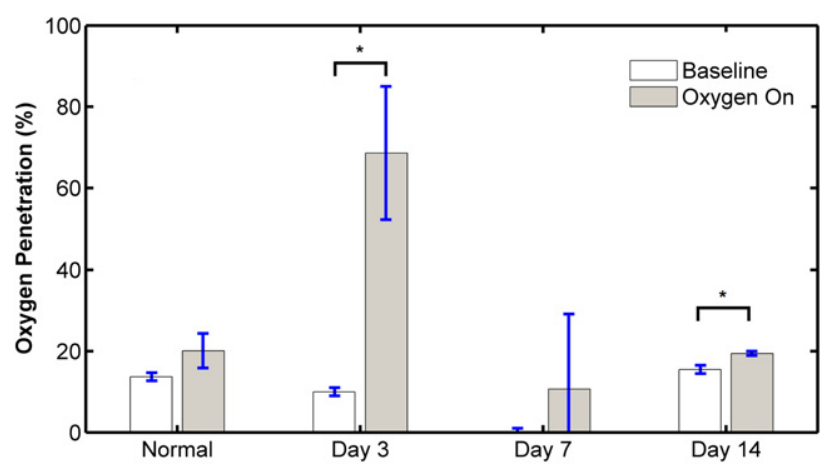

Figure 5. In vivo tissue oxygen penetration was quantified by a fiber optic oxygen probe. By inserting the fiber optic probe beneath the skin and measuring before and after 100\% oxygen was introduced, we recorded elevated oxygen tensions of $20,70,10$, and $20 \%$ for normal, days 3, 7, and 14 after wounding tissues, respectively, compared with their baselines $(p=0.121, p=0.025, p=0.423$, and $p=0.006)$. Day 7 , representing wounds with distinct scabs, gave inconsistent results but showed overall low oxygen permeability. Error bars denote standard deviations, $n=3$ wounds. increments) over time was measured and displayed in Figure 4. At each time point (1, 2, 3, 5, 10 minutes), as the thickness, i.e., depth, of the phantom tissue increased, the oxygen concentration through that depth decreased. However, over time the oxygen at all depths equilibrated up to a final concentration. After 10 minutes of $100 \% \mathrm{O}_{2}$ delivery, concentration across $0.8 \mathrm{~mm}$ thickness was $78 \pm 5 \% \mathrm{O}_{2}$.

\section{In vivo tissue oxygen penetration}

The extent of wound oxygen penetration was measured using the fiber optic oxygen probe. Without gas, baseline oxygen as a combination of ambient diffusion and skin perfusion measured from 10 to $15 \%$ for all conditions except day 7 (scabbing with inconsistent and low oxygen permeability). With $100 \%$ oxygen delivered to the bandage, normal tissue and wound tissues at days 3, 7, and 14 after wounding measured $20 \%, 70 \%, 10 \%$, and $20 \%$, respectively. This time-varying penetration corresponds to thinner tissue shortly after wounding, thicker and less permeable tissue as fibrin clot maturation occurs at day 7 , and a return to post-closure tissue that resembles normal skin. In summary, measurements at days 3 and 14 showed that the oxygen bandage indeed delivered significantly elevated $(p=0.025$ and $p=0.006$, respectively; Figure 5) oxygen tensions to the wound tissues, while day 7 with mature fibrin clot did not, $p=0.423$.

\section{Wound closure}

Preliminary results (data not shown) indicated that application of a microfluidic bandage itself with no oxygen delivery had no effect on normal wound closure. For the actual experiment, wound area was used as the end point for each of the 14 time points. ANOVA did not reveal a significant group effect, $p=0.43$. As expected, diabetic wounds closed more slowly than nondiabetic wounds $(p=0.09, \mathrm{CI}=[-3.48,15.46])$, as shown in Figure 6. No difference in closure rate was seen between oxygen-treated and nontreated diabetic wounds, $p=0.73$.

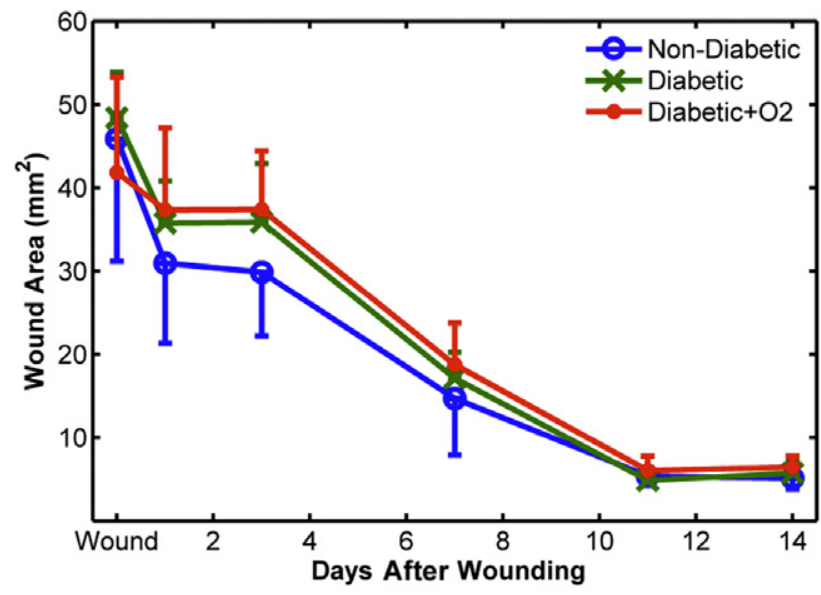

Figure 6. Oxygen bandage treatment does not accelerate or slow external wound closure in diabetic mice. Error bars denote standard deviations, $n=5$ wounds. 

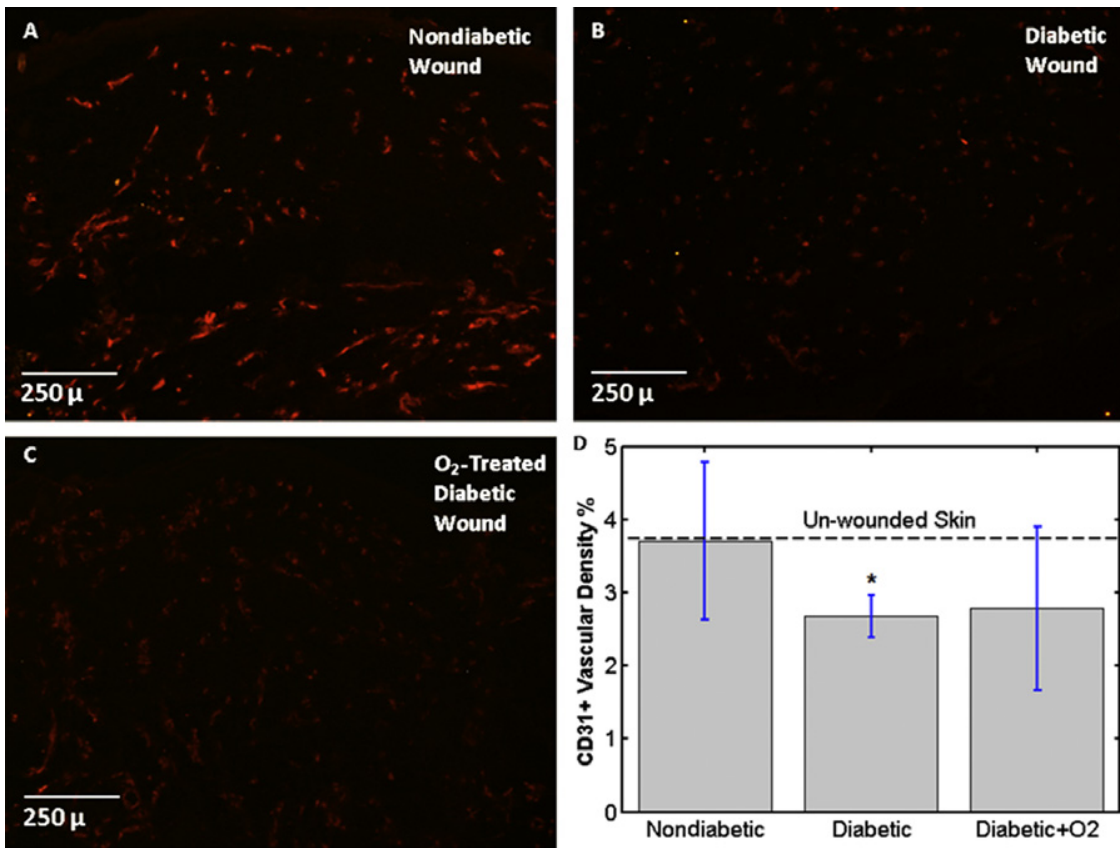

Figure 7. Staining shows extent of angiogenesis in (A) nondiabetic, (B) diabetic, and (C) oxygen-treated diabetic wounds with red color corresponding to CD31 positive areas. (D) Nondiabetic wounds have the same density of CD31 positive areas as unwounded skin while diabetic nontreated wounds have lower density, $p=0.036$. Oxygen-treated diabetic wounds, though marginally higher but not significantly different than the other groups. Error bars depict standard deviations, $n=5$ wounds.

\section{CD31 quantification of angiogenesis}

To examine the extent of angiogenesis beneath the surface of the closing wound, quantification of CD31 staining was performed on specimens derived at 14 days after wounding. Measurements were done in the wound bed of the section. ANOVA did not reveal a significant group effect, $p=0.19$. Higher levels of CD31 positive staining were seen in normal wounds when compared with diabetic wounds $(p=0.036$, $\mathrm{CI}=[-0.11,2.17])$, as shown in Figure 7. CD31 staining was

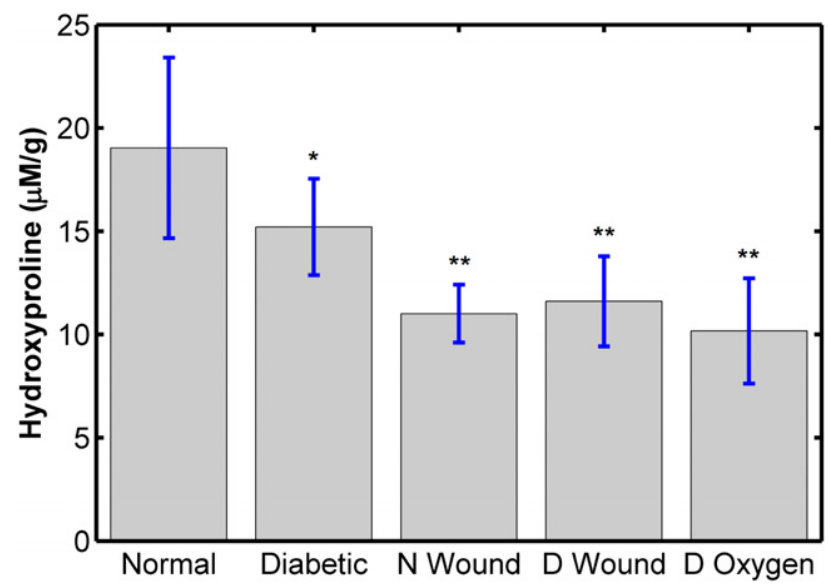

Figure 8. Hydroxyproline shows lower collagen content in unwounded diabetic compared with normal skin $\left({ }^{*} p=0.06\right)$. All 14 days wounded skins have lower collagen content (** $p<0.01)$ and are not statistically different from each other (normal, diabetic, normal wound, diabetic wound, diabetic wound treated with oxygen). Blue error bar denotes standard deviation, $n=5$ wounds. marginally higher in oxygen-treated wounds but was not significantly different between treated and nontreated diabetic wounds, $p=0.83$. Overall, the analysis of wound capillary content suggested no significant influence of oxygen bandage on angiogenesis in wounds of diabetic mice.

\section{Hydroxyproline and collagen maturity}

To examine the extent and maturity of collagen deposition beneath the surface of the closing wound, hydroxyproline quantification and picrosirius staining were done on tissues harvested 14 days after wounding. Hydroxyproline was assayed immediately after tissue harvest to quantify the total amount of collagen deposited, without distinction of maturity. ANOVA found a significant group effect, $p=0.000$. Hydroxyproline quantification showed that at 14 days post wounding, the total content of collagen compared with normal nonwounded tissues was lower in all wounds, normal wounds $(p=0.0023, \quad \mathrm{CI}=[-12.77,-3.30]), \quad$ diabetic wounds $(p=0.0047, \quad \mathrm{CI}=[-12.47,-2.39])$, and treated diabetic wounds $(p=0.0022, \mathrm{CI}=[-14.09,-3.64])$ as shown in Figure 8. Furthermore, there was a marginal decrease in nonwounded diabetic vs. normal tissue, $(p=0.06, \mathrm{CI}=[-8.94$, 1.28]). However, no statistical difference was seen between any of the wounded samples.

Total collagen content does not provide a measure of collagen maturity, a feature that is known to be critical to the development of wound strength. ${ }^{35}$ To examine collagen maturity, picrosirius red staining and analysis was performed. Measurements were made in the wound bed of the section. ANOVA revealed a significant group effect, $p=0.000$. As expected, the ratio of red (mature)to green (immature) fibers under picrosirius red staining was higher in normal vs. nontreated diabetic wounds $(p=0.0005, \mathrm{CI}=[0.89,1.73])$ as shown in Figure 9. Importantly, oxygen-treated diabetic wounds exhibited significantly higher red to green ratios than 

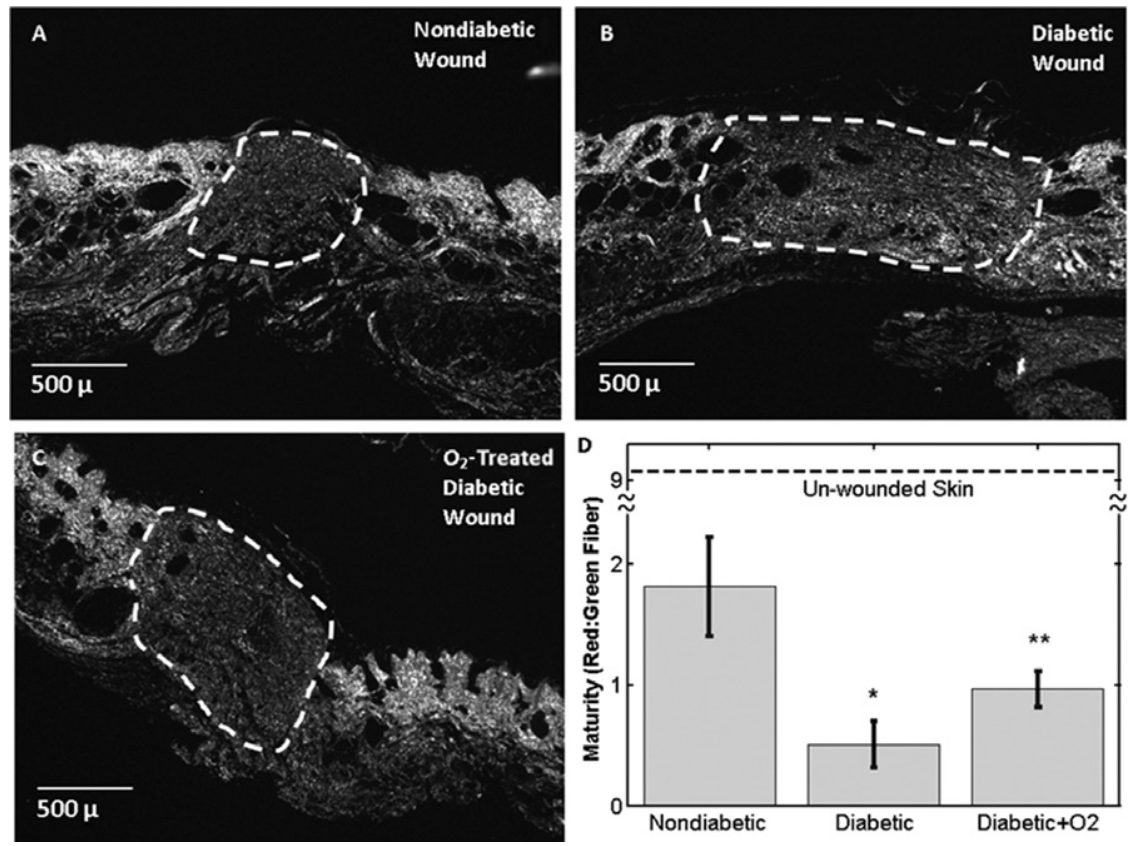

Figure 9. Picrosirius staining of (A) nondiabetic, (B) diabetic, and (C) oxygen-treated diabetic wounds shows the extent of collagen maturation, (D) maturity, expressed as ratio of red to green fibers, is lower in diabetic wounds, ${ }^{*} p=0.0005$, but improved with oxygen-treated diabetic wounds, ${ }^{*} p=0.0010$. Unwounded skin has much higher maturity ration at $9.1 \pm 4.2$. White dashed line denotes wound boundary. Blue error bars denote standard deviations, $n=5$ wounds. nontreated diabetic wounds $(p=0.0010, \mathrm{CI}=[0.22,0.70])$. While oxygen-treated wounds of diabetic mice showed greater collagen maturity than untreated diabetic wounds, the levels remained lower than those of wounds from normal mice, $(p=0.0012, \mathrm{CI}=[-1.30,-0.40])$. These data suggest collagen maturation, but not total collagen levels, is improved by the oxygen bandages.

\section{Limitations of the study}

The characterization of the oxygen bandage's effect on collagen maturation is subject to several limitations. First, we measured the oxygen transport through the tissue thickness via FOXY probe, which is sensitive to both gaseous and dissolved oxygen concentrations. Further histological tests, e.g., using hypoxyprobe, could compliment the measurement by showing tissue interactions with oxygen. Second, we used collagen maturation in our circular wounds as an indirect measure of tissue strength, as opposed to direct tensiometer measurement, which requires a linear wound. This is because diffusion is more symmetrical and predictable in a circular geometry, compared with a linear one. Lastly, the histological and tissue sample tests were done at day 14. Although this timing is consistent with collagen remodeling in $8-\mathrm{mm}$ wounds, it could be complimented by additional dynamical data from other time points. Despite these limitations, we have demonstrated oxygen penetration across the tissue thickness using the bandage, with a corresponding improvement in wound collagen maturation.

\section{DIsCUSSION}

Our studies describe the development of a novel oxygen delivery method through the construction of a microfluidic bandage. Prior to this oxygen bandage, the only means of oxygen therapy for wounds are hyperbaric chamber and topical bladders. The oxygen bandage through its diffusion membrane delivers oxygen conformally to the wound without convective drying or high pressure of previous devices. In addition, unlike oxygen bladders, the boundary layer of oxygen diffusion is eliminated due to its direct application to the wound resulting in more thorough oxygenation. Because of this ability we were able to see modulations in the wound collagen maturity underneath the surface. Moreover, oxygen bandages can be deployed with small medical grade gas supply to provide portability and convenience compared with hyperbaric chamber or topical oxygen bladders.

Using the bandage, we applied oxygen modulation to $8 \mathrm{~mm}$ diabetic murine wounds. Diabetic wounds are hypoxic, ${ }^{19,36-39}$ and the larger wound size has longer closure, which can better illustrate the improvements of wound healing. Up to $70 \%$ (prior to day 7) oxygen can penetrate across the wound thickness. External closure did not show significant difference between oxygen-treated and nontreated diabetic wounds. Similarly, CD31 staining showed marginal but not significantly higher angiogenesis in oxygen-treated diabetic wounds than nontreated ones. Although total content of collagen was not significantly different among the three groups, collagen maturity was significantly improved in oxygen-treated diabetic wounds vs. nontreated ones. Collagen maturation involves a transition from Type III to Type I fibers and cross-linking of immature fibers, and has been shown to be heavily dependent on oxygen partial pressure. ${ }^{12,14}$ Maturation of collagen leads to stronger tissue strength, resulting in a better healing wound that is less likely to reopen. Our results demonstrate that the diffused oxygen does provide improved healing underneath the epidermis in terms of collagen maturation, notwithstanding the marginally different angiogenesis and similar closure rates.

Beyond oxygen itself, one additional consideration is that alternative procollagen and angiogenic pathways such as 
lactate signaling and/or growth factor production by inflammatory cells may also be functioning. ${ }^{40}$ Despite being associated with hypoxic conditions, recent studies on oxygendependent lactate modulation of vascular endothelial growth factor and collagen suggested that lactate participates in a feed-forward amplification of wound healing cues. As lactate is abundantly produced by inflammatory cells, it may be that the oxygen bandage has evoked changes in the inflammatory response, a situation that may have modulated angiogenesis on top of hyperoxia-enhanced collagen synthesis, considering the high oxygen penetration we measured at day 3 .

Results using the oxygen bandage provide a comparison to existing devices. HBOT wound therapy has shown improved closure, more angiogenesis and collagen maturity. Its mechanism is well-understood to include a systematic elevation of oxygen tension in blood and plasma. On the other hand, previous studies using TOT showed inconsistent effects. Our results suggest that the localized, moisture-stable oxygen bandage could deliver up to $70 \%$ (prior to day 7) oxygen across the wound thickness and improve collagen maturation underneath. Whether this effect involves elements of epithelial-fibroblast cross talk remains to be examined. In any case, because of the ease and scalability of the oxygen bandage technique, larger studies that provide more information about effective dosages and mechanisms of action can now be conceived.

Future implementations of the oxygen bandages could provide additional temporal modulation of oxygen through the use of pneumatic valves. ${ }^{41}$ Additionally, the concept of topical delivery through controlled diffusion can be applied to other therapeutic agents well beyond oxygen. Cytokines, growth factors, metabolic factors like lactate, and even cellladen hydrogels can be administered using microfluidic mechanisms. Microfluidic bandages therefore provide a novel delivery platform that will enable many future wound healing experiments.

In summary, we have demonstrated a microfluidic oxygen bandage that can be used to locally deliver oxygen to the wounds. It can deliver oxygen concentrations from 0 to $100 \%$ in 60 seconds rise time, providing conformal coverage to the wound, and penetrate wound tissues as measured in agar phantom and in vivo wounds. Treatment of murine diabetic wounds with this microfluidic oxygen bandage improved healing outcomes in the form of higher collagen maturity. These findings suggest that the microfluidic oxygen bandage may be a useful technique for studying poorly healing wounds.

\section{ACKNOWLEDGMENTS}

This work was supported by the Multidisciplinary Oral Sciences Training Program, National Institutes of Health Grants T32 DE018381, R01 GM050875, and NSF DBI0852416.

Conflict of Interest: There are no conflicts of interests for all of the authors on this manuscript.

\section{REFERENCES}

1. Niethammer P, Grabher C, Look T, Mitchison TJ. A tissue-scale gradient of hydrogen peroxide mediates rapid wound detection in zebrafish. Nature 2009; 459: 996-9.
2. Shreeniwas R, Koga S, Karakurum M, Pinsky D, Kaiser E, Brett J, Wolitzky BA, Norton C, Plocinski J, Benjamin W. Hypoxiamediated induction of endothelial cell interleukin-1 alpha. An autocrine mechanism promoting expression of leukocyte adhesion molecules on the vessel surface. J Clin Invest 1992; 90: 2333-9.

3. Ceradini DJ, Gurtner GC. Homing to hypoxia: HIF-1 as a mediator of progenitor cell recruitment to injured tissue. Trends Cardiovasc Med 2005; 15: 57-63.

4. Knighton DR, Hunt TK, Scheuenstuhl H, Halliday BJ, Werb Z, Banda MJ. Oxygen tension regulates the expression of angiogenesis factor by macrophages. Science 1983; 221: 1283-85.

5. Pugh CW, Ratcliffe PJ. Regulation of angiogenesis by hypoxia: role of the HIF system. Nat Med 2003; 9: 677-84.

6. Crowther M, Brown NJ, Bishop ET, Lewis CE. Microenvironmental influence on macrophage regulation of angiogenesis in wounds and malignant tumors. J Leukoc Biol 2001; 70: 478-90.

7. O'Toole EA, Marinkovich MP, Peavey CL, Amieva MR, Furthmayr H, Mustoe TA, Woodley DT. Hypoxia increases human keratinocyte motility on connective tissue. J Clin Invest 1997; 100: 2881-91.

8. Ridgway PF, Ziprin P, Peck DH, Darzi AW. Hypoxia increases reepithelialization via an $\alpha v \beta 6$-dependent pathway. Wound Repair Regen 2005; 13: 158-64.

9. Murrell GA, Francis MJ, Bromley L. Modulation of fibroblast proliferation by oxygen free radicals. Biochem J 1990; 265 : 659-65.

10. Bradley TR, Hodgson GS, Rosendaal M. The effect of oxygen tension on haemopoietic and fibroblast cell proliferation in vitro. J Cell Physiol 1978; 97: 517-22.

11. Ikeda S, Yamaoka-Tojo M, Hilenski L, Patrushev NA, Anwar GM, Quinn MT, Ushio-Fukai M. IQGAP1 regulates reactive oxygen species-dependent endothelial cell migration through interacting with Nox2. Arterioscler Thromb Vasc Biol 2005; 25: 2295-300.

12. Hunt TK, Pai MP. The effect of varying ambient oxygen tensions on wound metabolism and collagen synthesis. Surg Gynecol Obstet 1972; 135: 561-7.

13. Gibson DR, Angeles AP, Hunt TK. Increased oxygen tension on wound metabolism and collagen synthesis. Surg Forum 1997; 48: 696-9.

14. Fu MX, Knecht KJ, Thorpe SR, Baynes JW. Role of oxygen in cross-linking and chemical modification of collagen by glucose. Diabetes 1992; 41 (Suppl. 2): 42-8.

15. Ohkubo Y, Kishikawa H, Arakia E, Miyata T, Isami S, Motoyoshi S, Kojima Y, Furuyoshi N, Shichiri M. Intensive insulin therapy prevents the progression of diabetic microvascular complications in Japanese patients with non-insulin-dependent diabetes mellitus: a randomized prospective 6-year study. Diabetes Res Clin Pract 1995; 28: 103-17.

16. Lerman OZ, Galiano RD, Armour M, Levine JP, Gurtner GC. Cellular dysfunction in the diabetic fibroblast: impairment in migration, vascular endothelial growth factor production, and response to hypoxia. Am J Pathol 2003; 162: 303-12.

17. Soriano FG, Virág L, Jagtap P, Szabó E, Mabley JG, Liaudet L, Marton A, Hoyt DG, Murthy KGK, Salzman AL, Southan GJ, Szabó C. Diabetic endothelial dysfunction: the role of poly(ADPribose) polymerase activation. Nat Med 2001; 7: 108-13.

18. Goren I, Kämpfer H, Podda M, Pfeilschifter J, Frank S. Leptin and wound inflammation in diabetic ob/ob mice: differential regulation of neutrophil and macrophage influx and a potential role for the scab as a sink for inflammatory cells and mediators. Diabetes 2003; 52: 2821-32. 
19. Galiano RD, Tepper OM, Pelo CR, Bhatt KA, Callaghan M, Bastidas N, Bunting S, Steinmetz HG, Gurtner GC. Topical vascular endothelial growth factor accelerates diabetic wound healing through increased angiogenesis and by mobilizing and recruiting bone marrow-derived cells. Am J Pathol 2004; 164 : 1935-47.

20. Baynes JW. Role of oxidative stress in development of complications in diabetes. Diabetes 1991; 40: 405-12.

21. Jeffcoate WJ, Harding KG. Diabetic foot ulcers. Lancet 2003; 361: 1545-51.

22. Tibbles PM, Edelsberg JS. Hyperbaric-oxygen therapy. $N$ Engl J Med 1996; 334: 1642-8.

23. Roeckl-Wiedmann I, Bennett M, Kranke P. Systematic review of hyperbaric oxygen in the management of chronic wounds. $\mathrm{Br} \mathrm{J}$ Surg 2005; 92: 24-32.

24. Sanders AP. Succinate: protective agent against hyperbaric oxygen toxicity. Science 1965; 150: 1830-31.

25. Said HK, Hijjawi J, Roy N, Mogford J, Mustoe T. Transdermal sustained-delivery oxygen improves epithelial healing in a rabbit ear wound model. Arch Surg 2005; 140: 998-1004.

26. Kalliainen L, Gayle GM, Schlanger R, Sen C. Topical oxygen as an adjunct to wound healing: a clinical case series. Pathophysiology 2003; 9: 81-7.

27. Gordillo GM, Roy S, Khanna S, Schlanger R, Khandelwal S, Phillips G, Sen CK. Topical oxygen therapy induces vascular endothelial growth factor expression and improves closure of clinically presented chronic wounds. Clin Exp Pharmacol Physiol 2008; 35: 957-64.

28. Fries RB, Wallace WA, Roy S, Kuppusamy P, Bergdall V, Gordillo GM, Melvin WS, Sen CK. Dermal excisional wound healing in pigs following treatment. Mutat Res 2005; 579: 172-81.

29. Fischer BH. Topical hyperbaric oxygen treatment of pressure sores and skin ulcers. Lancet 1969; 294: 405-9.

30. Vollmer AP, Probstein RF, Gilbert R, Thorsen T. Development of an integrated microfluidic platform for dynamic oxygen. Lab Chip 2005; 5: 1059-66.

31. Lo JF, Sinkala E, Eddington DT. Oxygen gradients for open well cellular cultures via microfluidic substrates. Lab Chip 2010; 10 : 2394-401.
32. Oppegard SC, Nam K, Carr JR, Skaalure SC, Eddington DT. Modulating temporal and spatial oxygenation over adherent cellular cultures. PLOS ONE 2009; 4: e6891.

33. Matthies AM, Low QEH, Lingen MW, DiPietro LA. Neuropilin-1 participates in wound angiogenesis. Am J Pathol 2002; 160: 289-96.

34. Saville DJ. Multiple comparison procedures: the practical solution. Am Stat 1990; 44: 174-80.

35. Levenson SM, Geever EF, Crowley LV, Oates JF, Berard CW, Rosen H. Healing of rat skin wounds. Ann Surg 1965; 161: 293-308.

36. Niinikoski JHA. Clinical hyperbaric oxygen therapy, wound perfusion, and transcutaneous oximetry. World J Surg 2004; 23 : 307-11.

37. Abidia A, Laden G, Kuhan G, Johnson BF, Wilkinson AR, Renwick PM, Masson EA, McCollum PT. The role of hyperbaric oxygen therapy in ischaemic diabetic lower extremity ulcers: a double-blind randomised-controlled trial. Eur J Vasc Endovasc Surg 2003; 25: 513-8.

38. Botusana IR, Sunkaria VG, Savua O, Catrinad AI, Grünlera J, Lindberga S, Pereirae T, Ylä-Herttualaf S, Poellingere L, Brismara K, Catrinaa SB. Stabilization of HIF-1 $\alpha$ is critical to improve wound healing in diabetic mice. Proc Natl Acad Sci U S A 2008; 105: 19426-31.

39. Sivan-Loukianovaa E, Awada OA, Stepanovica V, Bickenbachb J, Schattemana GC. CD34+ blood cells accelerate vascularization and healing of diabetic mouse skin wounds. J Vasc Res 2003; 40: 368-77.

40. Trabold O, Wagner S, Wicke C, Scheuenstuhl H, Hussain MZ, Rosen N, Seremetiev A, Becker HD, Hunt TK. Lactate and oxygen constitute a fundamental regulatory mechanism in wound healing. Wound Repair Regen 2003; 11: 504-9.

41. Lo JF, Wang Y, Blake A, Yu G, Harvat TA, Jeon H, Oberholzer J, Eddington DT. Islet preconditioning via multimodal microfluidic modulation of intermittent hypoxia. Anal Chem 2012; 84 1987-93. 